\title{
Optical efficiency characterization of LED phosphors using a double integrating sphere system
}

\author{
Paula Gorrotxategi, Marianne Consonni and Adrien Gasse
}

\author{
* Correspondence: \\ adrien.gasse@cea.fr \\ University Grenoble Alpes, F-38000, \\ France CEA-Leti, Minatec Campus, \\ F-38054 Grenoble, France
}

\begin{abstract}
We report on the methodology and implementation of a robust and accurate double integrating sphere system for measuring the absolute photoluminescence quantum yield and its temperature dependence of commercially available phosphors (garnets, silicates and nitrides). The potential of our instrument for the examination of light interaction with samples of different absorption and diffusion coefficients is also presented, as optical properties of luminescent materials have a major impact on the efficiency of LED's packaging. Our work gives special attention to the control and the optimization of light losses in the optical system in order to ensure reliable measurements. The YAG:Ce phosphor shows the highest luminescence quantum yield at $97 \%$ efficiency, the green silicate the lowest with $79 \%$ efficiency. Silicate phosphors show up to $15 \%$ loss of luminescence intensity when temperature is raised to $140^{\circ} \mathrm{C}$

Keywords: Photoluminescence; Rare-earth-doped materials; Light emitting diodes; Integrating spheres
\end{abstract}

\section{Background}

Phosphor down conversion is the most common strategy to achieve white light using blue Light Emitting Diodes (LED) and phosphors. Among the simplest and most commonly used methods, there is the use of the well-known yellow YAG:Ce phosphor (with a reputed high quantum efficiency) pumped by a blue (450-460 nm) wavelength source. This easy and efficient method produces a white LED with a color rendering index (CRI) of 70-80. Only a limited number of phosphors are known to be suitable as conversion materials due to the multiple requirements needed to fulfill this conversion task. Their excitation and emission spectra have to be of adequate broadness and energies in order to yield the required color rendering index and luminous efficiency. Phosphors also have to present a high fluorescent quantum yield and a high thermal stability. They should present a small particle size distribution and show a homogeneous spherical morphology in order to reduce scattering, to improve quantum yield and to be easily mixed in silicones, epoxy or other resin matrixes [1,2].

Accurate characterization of phosphors used in LED for Solid State Lighting (SSL) is essential to well understand the light extraction and conversion efficiencies of phosphor converted LED devices. Precise evaluation of photoluminescence (PL) efficiency of 
phosphors is not an easy task, mainly due to the difficulty of well understanding the behavior of reflected and transmitted light (diffuse and specular), as well as the need of precise calibration of light losses in the optical measurement system. When a light wave hits a silicone/phosphor film, several phenomena happen. The light is reflected at the surface (specular and diffuse behavior), selectively absorbed by the phosphor, scattered by phosphor particles (differently depending on the particle size), converted to different wavelengths or transmitted through the film. That is why the accurate understanding and characterization of processes describing light and phosphor particle interaction are not straightforward [3,4]. The quantum yield (QY) of a luminescent material is defined as the ratio of the number of photons emitted to the number of photons absorbed by the irradiated sample; it characterizes a radiative transition in combination with the luminescence lifetime, luminescence spectrum and photo stability of the phosphor.

Quantum yield is a selection criterion for luminescent materials used in solid state lighting applications. Knowledge of the QY provides a crucial feedback in the development of new synthesis techniques for the variety of luminescent materials being studied. Despite of the importance of accurate evaluation of luminescence quantum yields, very few studies addressing phosphor absolute QY values and measurement methodologies can be found in literature [3-5]. In these references, photoluminescence quantum yield value for the yellow YAG:Ce phosphor is often loosely defined as being higher than $90 \%$. Instrumentation can be a major source of variability on QY determinations, making difficult the comparison of fluorescence measurements between various instruments and over time [6]. Standardization can be achieved with reliable standards (solid standard samples are very rare) in combination with specific protocols for instrument characterization and instrument performance validation. Fluorescent signals may be influenced by both sample and instrument specific effects. To date, QY values of different phosphors (used in SSL) are addressed in literature [7], however the majority of these works give very little specifications (if any) on the procedures (and instrumentation) carried out for those QY evaluations. Moreover, the accuracy of the presented values is very rarely specified. A famous instrument manufacturer has launched onto the market a commercial instrument [8] able to measure absolute quantum yield values of solid and liquid luminescent materials based on a single integrating sphere system, where the sample is placed on the sphere wall and it is irradiated at a normal incidence. Incident and converted light are measured in reflection. The precision of this instrument is stated to be around 3\%. This commercial instrument presents excellent characteristics for absolute luminescent QY measurements, using a set-up with a single integrating sphere that holds the sample in the center of the sphere and also avoids trouble with light losses. However, the behavior of incident light when interacting with samples of different absorption and diffusion properties cannot be studied thoroughly, as reflected and transmitted light are not differentiated in a single-sphere set-up. A double integrating sphere system (DIS), where reflected and transmitted light are separately measured, will give more information on the light interaction with the phosphor: amount of reflected vs transmitted converted light, Yellow to Blue Ratio difference between reflected and transmitted light... These effects are driven by the physical properties of phosphors (absorbance, particle size or concentration). Thus, the analysis of both reflected and transmitted light properties leads to a deeper understanding of these phenomena, which is essential for optimizing LED 
package performances as they will have a remarkable impact on light extraction and spatial color distribution.

This work presents, for the first time, a detailed methodology on the measurements of absolute photoluminescence quantum yields (PL-QY) of solid phosphors carried out in a double integrating sphere system. We also show and emphasize the potential of this instrument for extensive studies on optical properties of luminescence materials such as phosphors, nano-crystals, QDs or turbid media, reinforcing the work of previous studies that implemented double integrating spheres systems [9-11]. We present a DIS instrument that is optimized for absolute PL-QY measurements. The integrating spheres have been designed to maximize their gain and light homogeneity within the sphere before reaching the detector (as fluorescence can be a very weak signal). The spectrometers (Ocean Optics, Maya Pro-2000) were also chosen for their low electronic noise, temperature stability and high dynamic range. A special effort was done to minimize light losses in the optical system in order to accurately evaluate absolute PL-QY values. To do so, glass slides, which are very commonly employed to hold the sample, were not used in order to avoid total internal reflection effects; the sample was irradiated with a small beam (relative to the sample port size, at least 2/10 ratio) due to the very diffusive nature of the phosphor samples; and finally, a method to trap the specular reflection from the sample's front face within the DIS system was applied.

Several commercially available phosphors used in SSL were chosen for our work, such as yellow YAG:Ce garnets $\left(\mathrm{Y}_{3} \mathrm{Al}_{5} \mathrm{O}_{12}: \mathrm{Ce}^{3+}\right)$, green, yellow and orange silicates $\mathrm{M}_{2} \mathrm{SiO}_{4}: \mathrm{Eu}^{2+}\left(\mathrm{M}=\mathrm{Ba}^{2+}, \mathrm{Sr}^{2+}, \mathrm{Ca}^{2+}\right)$ and red nitrides $\mathrm{M}_{2} \mathrm{Si}_{5} \mathrm{~N}_{8}\left(\mathrm{M}=\mathrm{Ca}^{2+}, \mathrm{Sr}^{2+}, \mathrm{Ba}^{2+}\right)$ [12]. Our choice for a double integrating sphere system, instead of a single sphere system, was driven by the fact that with our set-up, reflected and transmitted light from the sample can be simultaneously measured within the same experimental conditions and configuration. In this way, accurate measurements of absorption of blue incident light are available, together with the fraction of reflected and transmitted light, providing valuable optical constants of the studied sample [13]. A discussion on the self-absorption phenomena happening in integrating sphere systems is addressed in this article. We also studied the temperature dependence of luminescence intensity of these phosphors, as thermal stability is a big concern for high power light emitting diodes used in the lighting industry. Temperature studies are performed up to $140^{\circ} \mathrm{C}$, which is generally the highest recommended junction temperature in LED packages. Finally, an examination of incident blue light behavior when interacting with YAG:Ce thin film samples of different concentrations is presented.

\section{Methods}

The sample, placed between the two integrating spheres (6 inch diameter), is directly irradiated by a collimated blue beam at $450-460 \mathrm{~nm}$ wavelength. This wavelength was chosen as most of blue LED in the market emit at this range. Figure 1 shows a scheme of the DIS system. The first sphere, $\mathrm{R}$, measures the total light reflected from the sample (specular and diffuse) and the second one, $\mathrm{T}$, measures the total transmitted light (specular and diffuse). The contact between the two integrating spheres has to be excellent to avoid light losses in the system. Light is simultaneously measured by two Ocean Optics (Maya Pro-2000) spectrometers able to span a large wavelength 


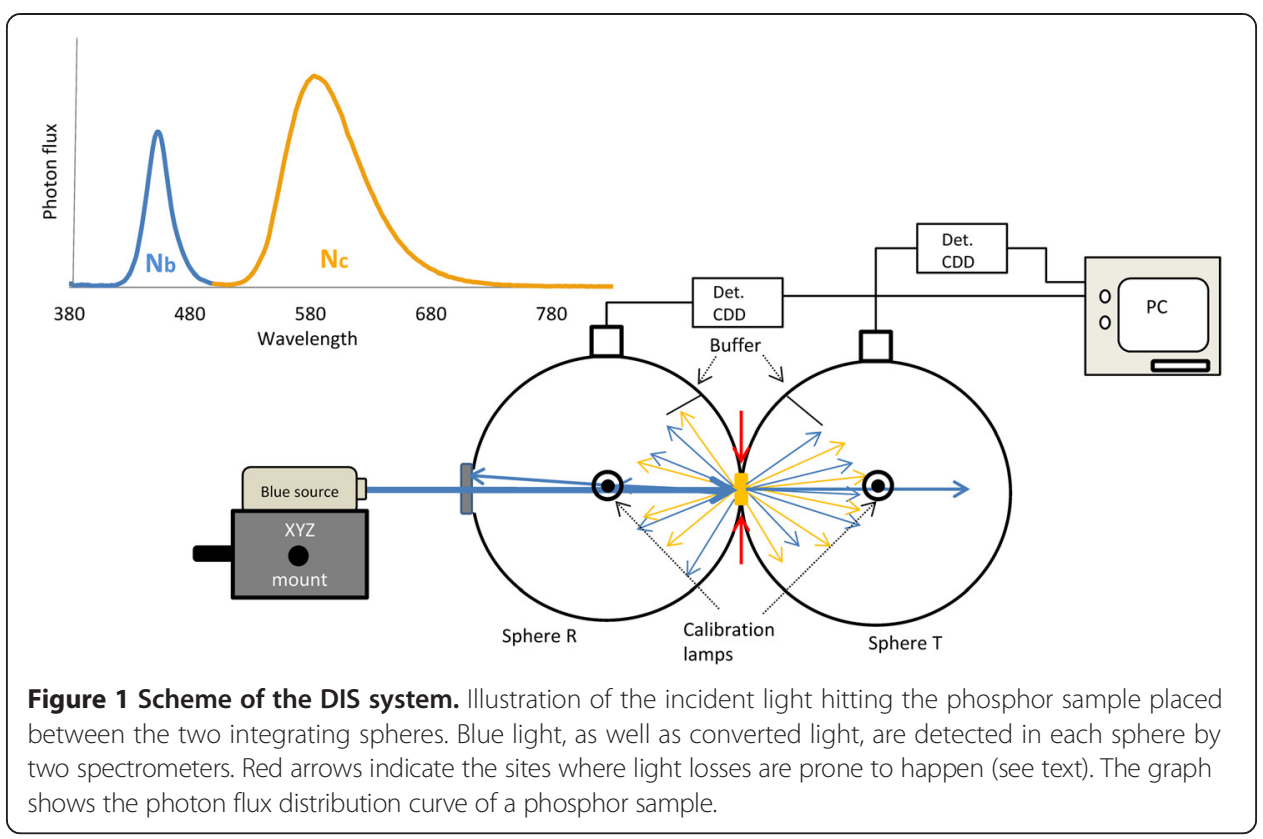

range $(250-1100 \mathrm{~nm})$ with $0.5 \mathrm{~nm}$ resolution intervals. The spectrometers are connected to the spheres by fibers, in which diffusers are connected to improve light detection. These have a viewing angle of $\pm 13^{\circ}$, which is extended to a hemispherical field through the diffusers and allows detecting the entire sphere signal. The baffle avoids direct illumination onto the detector. Two silicon-based back-thinned, 2D FFT-CCD uncooled low-noise detectors (Hamamatsu S10420) are used to measure the integrated radiant flux in each sphere. By using a back-thinned CCD as the detector, the sensitivity for spectral detection in both, the short and long wavelength regions, is greatly improved compared with that of an optical detection system that uses a conventional photo detector [5].

The total luminous flux inside the spheres is calibrated using the radiant flux from Quartz Tungsten Halogen (QHT) lamp irradiance standards that are attached to the spheres. One lamp is located in sphere $\mathrm{R}$ and the other one in sphere T. These lamp standards (supplied by LabSphere) include a calibration certificate and a calibrated spectral flux data, in $\mathrm{W} / \mathrm{nm}$. The calibration of each sphere is done individually by introducing a Spectraflect sample (same material that covers the wall of the sphere) in the sample port and switching on one calibration QHT lamp at a time. This calibration is necessary to well account for the measured spectral flux within each sphere. A home-made LabView 2010 interface permits real time data acquisition and analysis: excitation and emission spectra of the studied sample $(\mu \mathrm{W} / \mathrm{nm})$, integrated spectral flux in $\mathrm{R}$ and $\mathrm{T}$ spheres, absolute PL quantum yields, colorimetric coordinates, CRI... are some of the most advantageous features provided by this acquisition interface.

Equation 1 shows the experimental parameters needed to calculate absolute QY values. Total number of converted photons $\mathrm{N}_{C}$ is the sum of reflected converted photons $\mathrm{N}_{\mathrm{CR}}$ and transmitted converted photons $\mathrm{N}_{\mathrm{CT}}$. $\mathrm{N}_{\mathrm{B}}{ }^{\text {Abs }}$ states for absorbed blue photons. Total number of non-absorbed blue photons is divided into reflected blue photons $\mathrm{N}_{\mathrm{BR}}$ and transmitted blue photons $\mathrm{N}_{\mathrm{BT}}$. $\mathrm{N}_{\mathrm{B}}{ }^{\text {Inc }}$ is the total number of photons incident on the phosphor sample. 


$$
Q Y=\frac{N_{C}}{N_{B}^{A b s}}=\frac{N_{C R}+N_{C T}}{N_{B}^{I n c}-\left(N_{B R}+N_{B T}\right)}
$$

An integrating sphere is characterized by its diameter, its ports, its baffle and its wall reflectance (typically $\sim 98 \%$ ). The baffle is normally located between the sample and the detector, to ensure that all light reflected by the sample is uniformly distributed across the sphere walls before hitting the detector. In a double integrating sphere configuration, for a given sample port size diameter, the smaller the beam diameter relative to the sample port diameter, the fewer the light losses in the optical system and, hence, the more accurate the experimental measurement [13]. The wall surface of our integrating spheres was coated with commercial standard reference Spectraflect ${ }^{\oplus}$ material (from LabSphere supplier) which has a constant reflectivity $(\mathrm{R}=98 \%)$ within the wavelength range of interest (400-800 $\mathrm{nm}$ ).

Due to the sample holder capacity, sample dimensions were limited to planar films of $18 \times 13 \mathrm{~mm}^{2}$ surface and $2 \mathrm{~mm}$ thickness. In these experiments, $0.5 \mathrm{~mm}$ thick films of phosphor mixed in a two-part optical silicone optical encapsulant were used. Thicker samples were not made in order to avoid light losses in the DIS system [13]. As the thickness of the sample increases, more light is likely to leave from the edges of the sample and get lost by hitting non-reflecting surfaces. In addition, the radial path lengths are nearly a factor of four greater in a $2 \mathrm{~mm}$ sample compared to a $0.5 \mathrm{~mm}$ sample, and photons will encounter four times more scattering events, enhancing the probability of light losses. To prepare the films, a silicone $(\mathrm{n}=1.41$ @ $450 \mathrm{~nm})$ was mixed with different phosphor powders. Once the phosphor added, the mixture was well mixed and homogenized. Then, the phosphor/silicone mixture was degased in a vacuum oven at ambient temperature for 15-20 min. When all air bubbles were eliminated, the mixture was dispensed between glass slides. After 10 min curing at $150^{\circ} \mathrm{C}$, the phosphor/silicone film was carefully removed from the slides.

The sample, placed between the two integrating spheres, was illuminated by a blue laser module ( $450 \mathrm{~nm}, \mathrm{FWHM}=5 \mathrm{~nm}, 12 \mathrm{~mW})$. This laser source substituted a collimated LED source (Thorlabs M-455 L3, FWHM = $20 \mathrm{~nm}$ ) primarily used in the experiment. Several problems were found when implementing a LED as the illumination source. To begin with, it was difficult to get a good quality collimated beam small enough and powerful enough for our experimental requirements. The $8 \mathrm{~mm}$ diameter collimated beam was still too large relative to the $10 \mathrm{~mm}^{2}$ surface of the sample port size not to induce light losses in the DIS system: scattering light was likely to hit nonreflecting surfaces and get lost when escaping from the four edges of the sample. In addition, when using a LED illumination source, specular Fresnel losses (equation 2) of the incident blue light induced by the surface of the silicone/phosphor film could not be accurately calibrated: specular reflectance that escaped from the 1 inch diameter entrance port could not be measured.

$$
R_{1 F}=\left(\frac{n_{1}-n_{2}}{n_{1}+n_{2}}\right)^{2}
$$

Equation 2 represents the amount of blue incident light that is specularly reflected from the front surface of the silicone/phosphor thin sample. $\mathrm{n}_{2}$ states for the refractive index of silicone $\mathrm{n}=1.41$ at $450 \mathrm{~nm}$ and $\mathrm{n}_{1}$ is the air refractive index. The above- 
mentioned issues were completely avoided when a well collimated, $1.5 \mathrm{~mm}$ diameter laser beam was used; this beam was small enough to avoid rays escaping from the edges of the sample (beam size was small compared to the sample port size). Moreover, Fresnel losses were completely avoided as the 1 inch aperture of the integrating sphere was closed with a mechanical piece that let the $1.5 \mathrm{~mm}$ beam pass through (through a 5 $\mathrm{mm}$ whole) and recovered the reflected specular light within the $\mathrm{R}$ sphere. This piece was covered with Spectraflect ${ }^{\circ}$ material in order to provide a lambertian reflectance inside the sphere. The angle of incidence of the beam was slightly tilted from the normal incidence (approx. $3^{\circ}$ ) by careful adjustment of the position of the source installed on a XYZ optical mount. When the beam passes through the $5 \mathrm{~mm}$ whole, it hits the sample slightly off-axis and the reflected light encountered the aluminum piece that closed the 1 inch aperture of the sphere, getting trapped in the DIS system.

\section{Results and discussion}

Luminescence quantum yields

To determine absolute QY values of the commercial phosphors, first, the incident power (blue light) is measured with a standard Spectraflect ${ }^{\circ}$ sample in sphere R. Then, the Spectraflect ${ }^{\circ}$ sample is replaced by a phosphor/silicone sample, which is irradiated by the blue beam under the same experimental conditions. Absorption of the blue light by the silicone matrix was verified to be negligible for $0.5 \mathrm{~mm}$ thin films at $450 \mathrm{~nm}$. The spectrometer in $\mathrm{R}$ measures the reflected blue and converted spectral flux $(\mathrm{W} / \mathrm{nm})$ and the spectrometer in $\mathrm{T}$ measures the transmitted blue and converted spectral flux $(\mathrm{W} / \mathrm{nm})$. Then, spectral flux in $\mathrm{W} / \mathrm{nm}$ is converted to photons/nm. The number of photons is integrated over the blue excitation region ( 380-470 $\mathrm{nm}$ for the laser source and 380-490 $\mathrm{nm}$ for the LED source) and the emission wavelength range $(\sim 471-800 \mathrm{~nm}$ and $491-800 \mathrm{~nm}$ for the laser and LED source respectively) to obtain the total integrated photons, $N_{B}$ and $N_{\mathrm{C}}$. These values are used to evaluate absolute QY from Equation 1.

To ensure accurate response of the DIS system and therefore, correct values of PL-QYs, calibration of the dark current (baseline) noise of the spectrometers and of the luminous flux inside integrating spheres (using the QHT standard lamps) was done previous to a set of measurements. To avoid derives on spectrometer baseline during the measurements, the spectrometers were switched on $30 \mathrm{~min}$ before measurements started to ensure good signal baseline stabilization. Once the baseline level stabilized, the RMS noise (approx. 6 RMS counts) of the spectrometers was calibrated and their integration time was optimized for our working intensities, avoiding light saturation on the receptor. The reproducibility of the spectrometers (the stability of the baseline) was measured to be higher than $95 \%$ in $1 \mathrm{~h}$. However, calibration of the baseline signal was done every 10 minutes during measurements, as a preventive procedure.

The use of a stable laser source provides robustness during a whole set of measurements, thanks to its high power stability (0.5\%). However, every 20 minutes a reference measure (with a Spectraflect ${ }^{\circ}$ sample) was taken, to ensure an accurate calibration of the incident blue light. In addition, as the quality of the illuminated sample can drastically affect the emission intensity of the sample, big efforts were made to obtain homogeneous phosphor samples, without air bubbles and surface imperfections. 
Figure 2(a) shows the emission spectra of the green silicate, the yellow garnet (supplier A) and the red nitride measured in transmission, sphere T. Figure 2(b) illustrates the issue of the overlapping of the excitation and emission curves when a large bandwidth irradiation source is used, such as a LED.

Table 1 shows QY values of silicone/phosphors samples (with 20\% phosphor in weight) used in this study. This concentration was chosen as the density of phosphor particles in a $20 \%$ sample $\left(\sim 9 \times 10^{4}\right.$ particles $\left./ \mathrm{mm}^{3}\right)$ is of the same order of magnitude than the density of phosphor layers generally used in LED packages [2]. Errors on QY values are evaluated by calculating the standard deviation on the experimentally measured averaged QY values of 10 different measurements. This error gives an insight on the reproducibility of our instrument. Our biggest measurement imprecision comes from spectrometer performance that can be affected by some residual light "trapped" in the spectrometer (stray light), or random variations $(3 \%)$ on the dark current baseline due to temperature changes within its environment. Considering the high stability of the laser source, the good homogeneity of silicone/phosphor samples and the systematic calibration steps carried out before the measurements, we attribute the uncertainty of our measures to the spectrometers performance variability.

If the excitation source has a large spectral bandwidth (LED), the light spectrum of the source will overlap the emission photoluminescence spectrum of phosphors emitting at short wavelengths, effect that is notably seen on green phosphors. This effect leads to an additional error on the evaluation of quantum yield measurements. This issue is easily solved with the $451 \mathrm{~nm}$ laser module, as illustrated in Figure 2b. However, note that in real pc-LED packages, if green phosphors are used (usually in a phosphor blend), this overlapping area should be considered when evaluating the luminous efficiency of the whole LED package. Measurements were carried out by using both, the blue LED and the blue laser illumination sources. The enhanced values measured with the laser source make evident the need of a careful calibration and/or elimination of light losses in the instrument. The reliability of the laser based DIS system was proven by the agreement found on the measured QY value of the YAG:Ce phosphor and the value given by supplier A. Our values are also in fair agreement with phosphor QY values found in literature [14-16]. The differences between the QY values measured in this work
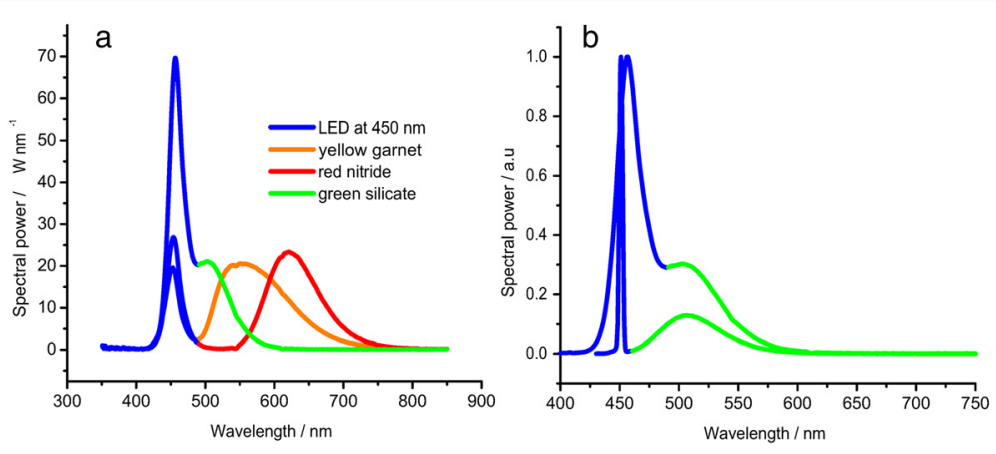

Figure 2 Emission spectra of phosphors at $\mathbf{4 5 0} \mathrm{nm}$ excitation wavelength. Graph a illustrates the emission spectra of a green silicate, yellow garnet and red nitride phosphors measured in the sphere T of the DIS system with blue LED excitation. Graph $\mathbf{b}$ shows the emission spectra of the green phosphor when it is illuminated either by a LED source or a monochromatic laser source. 
Table 1 Absolute quantum yield values of commercial phosphors

\begin{tabular}{|c|c|c|}
\hline Phosphor & Absolute-QY: LED@455 nm & Absolute-QY: laser@451 nm \\
\hline Yellow garnet* ${ }^{*}\left(\lambda_{\mathrm{em}}=558 \mathrm{~nm}\right)$ & $0.93 \pm 0.03$ & $0.96 \pm 0.02$ \\
\hline Yellow garnet ${ }^{* *}\left(\lambda_{\mathrm{em}}=558 \mathrm{~nm}\right)$ & $0.84 \pm 0.04$ & $0.87 \pm 0.03$ \\
\hline Green silicate* $\left(\lambda_{\mathrm{em}}=508 \mathrm{~nm}\right)$ & $0.75 \pm 0.04$ & $0.79 \pm 0.03$ \\
\hline Yellow silicate $*\left(\lambda_{\text {em }}=550 \mathrm{~nm}\right)$ & $0.84 \pm 0.03$ & $0.87 \pm 0.02$ \\
\hline Orange silicate* $\left(\lambda_{\mathrm{em}}=586 \mathrm{~nm}\right)$ & $0.88 \pm 0.03$ & $0.90 \pm 0.02$ \\
\hline $\operatorname{Red}_{\text {nitride }}^{*}\left(\lambda_{\mathrm{em}}=629 \mathrm{~nm}\right)$ & $0.89 \pm 0.04$ & $0.92 \pm 0.03$ \\
\hline
\end{tabular}

*Supplier A; **Supplier B. Errors represent the standard deviation $(2 \sigma)$ of the averaged QY value calculated for 10 measurements. QY values are obtained with a LED source at $456 \mathrm{~nm}$ and with a laser module at $451 \mathrm{~nm}$.

and the ones found in literature can be issued to the different engineering processes in phosphor fabrication or to inaccuracies on measurement procedures.

Our measurements have shown that the phosphor behaves identically under illumination of an incoherent LED source or a coherent laser source under our experimental conditions of low power irradiation (few tens of $\mathrm{mW}$ at the sample level, power density below $100 \mathrm{~mW} / \mathrm{cm}^{2}$ ). Fluorescence emission curves (shape and dominant emission wavelength) were identical under the LED or laser illumination for all phosphors. This gives evidence that molecular transitions and energy transfers remain identical under incoherent or coherent light illumination within this power regime. In addition, linear absorption regime (no saturation issues) was verified under radiation power lower than $50 \mathrm{~mW}$. Therefore we believe that under our experimental conditions the phosphor samples behave equally under the LED or the laser illumination. Thus, the intrinsic optical efficiency of the phosphor does not change under LED or laser illumination.

Another issue of the evaluation of QYs is related to self-absorption effects. Selfabsorption (SA) competes with the escape of the luminescence from the phosphor and can change the observed spectral shape, lifetime, and quantum yield from their true values. We consider two kinds of SA phenomena. First, a self-absorption that is an intrinsic property of phosphors and can be engineered by phosphor synthesis methods (the overlap area between their excitation and emission spectra will depend on the activator concentration and its homogeneous distribution on the crystalline structure [17]). Second, the SA which is due to the integrating sphere instrument, as the light emitted from the sample can be reabsorbed due to multiple reflections inside the sphere and the sample [6,18]. In this paper, the interpretation and evaluation of self-absorption phenomena is carried out following the methodology proposed by Ahn et al. [19], where the parameter $\boldsymbol{a}$ can be interpreted as the reabsorption probability of self-absorption of an emitted photon. It is evaluated using equation 3 [19], where the integrated emission spectral power of the "distorted" luminescent sample, $P_{d}\left(\lambda_{e m}\right)$, is compared to the "undisturbed" (without self-absorption, true spectra) integrated emission spectra of the same luminescent material $P\left(\lambda_{e m}\right)$. This undisturbed emission spectrum can be obtained using very diluted (low concentration: $3 \%$ ) samples. The observed quantum yield, termed here as $Q Y_{O b s}$, can be corrected for reabsorption effects with equation 4 [15], which subsequently leads to reabsorptioncorrected quantum efficiencies $Q Y_{C o r}$ 


$$
\begin{array}{r}
\int P_{d}\left(\lambda_{e m}\right) d \lambda_{e m} \\
a=1-\frac{\lambda_{\lambda_{e m}}}{\int_{e m}} P\left(\lambda_{e m}\right) d \lambda_{e m} \\
Q Y_{C o r}=\frac{Q Y_{O b s}}{1-a+a Q Y_{O b s}}
\end{array}
$$

Figure 3 shows the emission spectra of $0.5 \mathrm{~mm}$ thick film YAG:Ce samples at 3\%, $20 \%$ and $70 \%$ concentrations together with the emission curve given by the phosphor supplier (we assume that the emission curve given by the supplier is without selfabsorption effects induced by experimental procedures). Figure 3 shows an evident red shift on the emission curves of YAG:Ce phosphor samples when increasing sample concentrations from 3\% to $70 \%$. For $70 \%$ concentrated samples there is even a visible change on the shape of the curve. In addition, we measured that for the highly concentrated samples the QY is lower than the one measured with $20 \%$ concentrated samples. A QY of $93 \%$ was obtained for $70 \%$ concentrated samples, compared to $97 \%$ of the $20 \%$ concentrated samples. Reabsorption of high energy wavelengths of the emission spectra (and hence, re-emission at longer wavelengths) and non-radiative energy transfer to $\mathrm{Ce}^{3+}$ ions which emit at a slightly lower energy can explain the continuous red-shift with increasing phosphor concentration and the decrease on the photoluminescence quantum yield $[17,20]$.

If SA correction is applied by means of equation 3 to a $70 \%$ sample, a corrected QY value of $95 \%$ is obtained, instead of $93 \%$ obtained from the direct measurement. When applying equation 3 to a $20 \%$ sample in order to correctly evaluate the QY from sphere effects, a $Q Y_{\text {Cor }}$ value of $97.1 \%$ is obtained (against the $97 \%$ measured by the DIS), suggesting that SA effect plays a minor impact $(<1 \%)$ on the evaluated QY values in

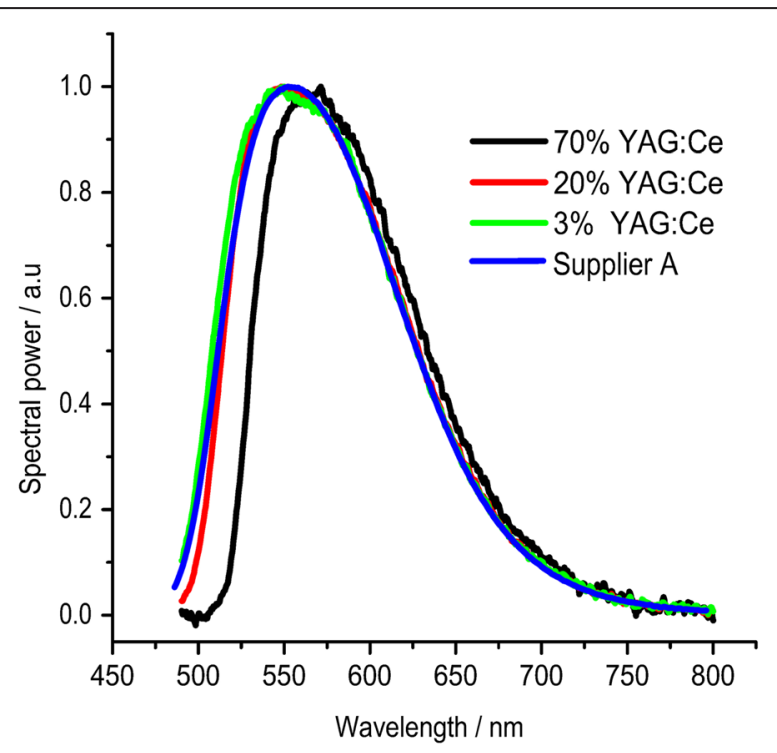

Figure 3 Red shift of the YAG:Ce emission curve induced by high phosphor concentration. The graph illustrates the measured emission spectra of $3 \%, 20 \%$ and $70 \%$ concentrated $0.5 \mathrm{~mm}$ phosphor films together with the emission curve given by the phosphor manufacturer (supplier A). 
our DIS system for $20 \%$ concentrated samples. Therefore, instrumental precision remains the largest source of error and results presented in Table 1 are proved to be accurate.

\section{Temperature stability measurements}

Phosphors luminescence is impacted by temperature and thermal quenching effects are known to degrade luminescence efficiency [20,21]. Thermal management is one of the biggest issues in high power white pcLED packages. Temperature dependence measurements on luminescence intensity of excited phosphors were carried out for silicone/ phosphor (20\%) films by heating them up to $140^{\circ} \mathrm{C}$. Temperature measurements were done in reflection using only the sphere R. A custom designed aluminum piece was attached to the sphere and phosphor films were deposited on its polished surface with the collimated blue beam irradiating them at a normal incidence. A NTC (Negative Temperature Coefficient) 10Kohm thermistor was placed on the aluminum piece, very close to the phosphor film $(<1 \mathrm{~mm})$ to ensure the accurate phosphor sample temperature. The aluminum piece was slowly heated using a heating adhesive device attached at its back side. Reflected light and converted light were continuously registered as temperature raised from $23^{\circ} \mathrm{C}$ to $140^{\circ} \mathrm{C}$ and emission intensity spectra were recorded at the different temperatures. Special care was taken to avoid direct contact of the hot aluminum piece with the Spectraflect ${ }^{\circ}$ material. No change in the integrating sphere response (gain), neither memory effects on the measured spectral flux were found after increasing the temperature up to $140^{\circ} \mathrm{C}$.

The results of these measurements are shown on the graphs illustrated in Figures 4 and 5. Silicate phosphors showed the largest temperature quenching effect with up to $15 \%$ of intensity loss at $140^{\circ} \mathrm{C}$ and a blue-shift on the emission curve [22], contrary to the garnet and nitride phosphors, that exhibit an excellent thermal stability at $140^{\circ} \mathrm{C}$ and a slightly red-shift at these high temperatures. Measurements were also carried out for a 70\% YAG:Ce concentrated phosphor and no difference was observed in thermal behavior in comparison to a $20 \%$ sample at $140^{\circ} \mathrm{C}$. Luminescence degradation has a

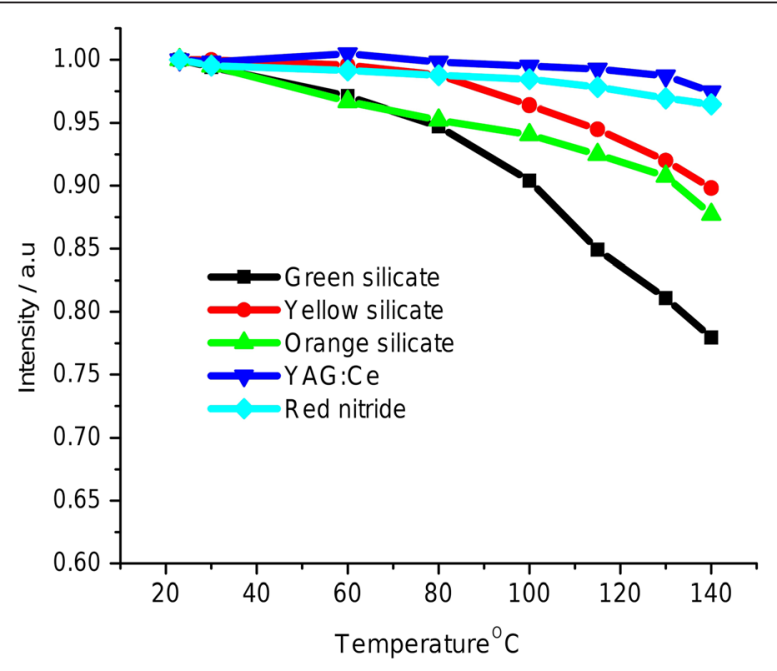

Figure 4 Luminescence intensity decay of the studied phosphors. 


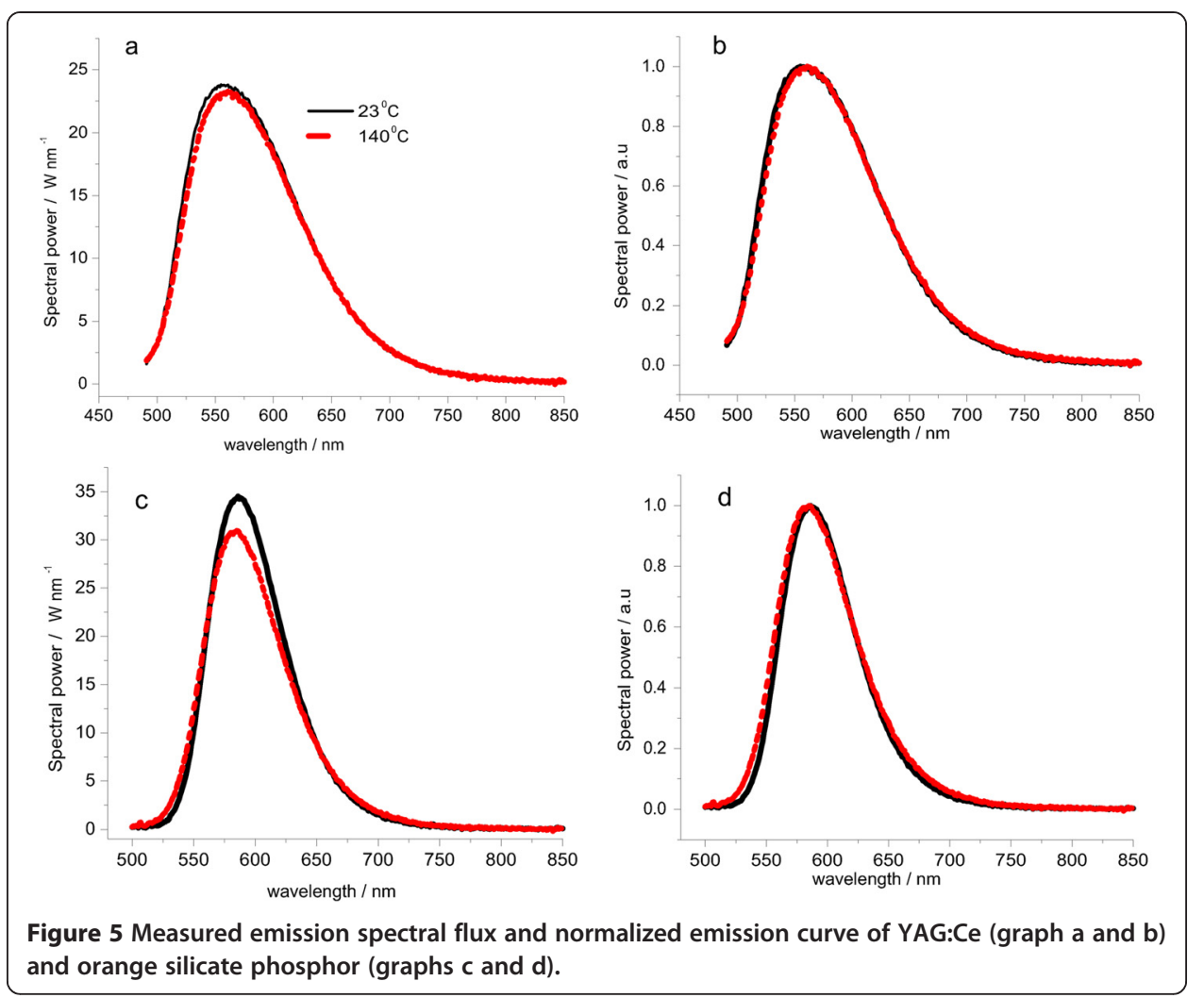

direct impact on the luminescence quantum yield. Absolute quantum yields were not measured with this "single-sphere" configuration, however the lower relative QY values measured at high temperature compared to those measured at room temperature (RT) with this one-sphere configuration, can be calibrated to absolute QY values measured at RT with the double sphere configuration. In Figure 4 the temperature dependence of the photoluminescence intensity for the phosphors studied in this work is presented. Absolute spectral power (graphs a and c) is illustrated to better evaluate the decay of the emission peak and normalized curves (graphs b and d) are shown to highlight the change of the curve shape of each phosphor with increasing temperature.

\section{Impact of sample concentration on light behavior}

One of the main goals of high power white LED development is to enhance and control light extraction (after phosphor conversion) from the packaging system (LED chip, phosphor layer, silicone encapsulate, reflector surface...). A study of light behavior interacting with phosphor samples of different concentration and particle size can be of great help for the development of a robust light extraction design for LED packages. We carried out some studies on light behavior using YAG:Ce phosphor samples at different concentrations (but same film thickness, $0.5 \mathrm{~mm}$ ), and the they are presented in Figure 6.

YAG:Ce phosphor presents high absorption of blue light, which causes rapid attenuation of transmitted blue and small reduction of reflected blue light with increasing phosphor concentration. The constant trend of the reflected (specular) blue light gives an idea of the reflectivity of silicone/phosphor samples. Reflected yellow light increases 


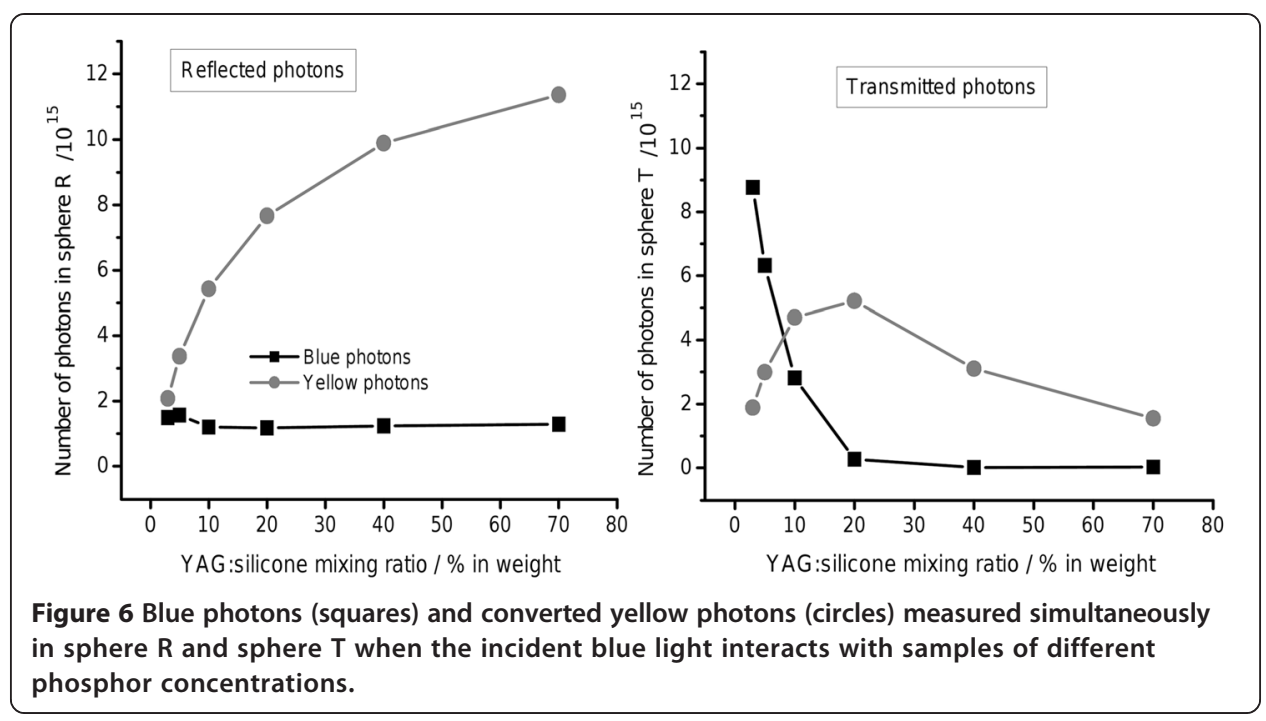

quickly as phosphor concentration is augmented. This trend was also found by Liu et al. [9] and it is evidenced by the graphs represented in Figure 6. Transmitted yellow light increases as the phosphor concentration increase, (as the incident blue light encounters more phosphor particles that make light conversion) until it reaches a maximum at about $20 \%$ concentration (in weight). At higher phosphor concentrations, transmitted yellow light decreases because an increase of the number of phosphor particles decreases the mean free path of the light (blue and converted) and obstructs its forward passage. Therefore, most of the converted light is backscattered while transmission of light drops. For $0.5 \mathrm{~mm}$ thick films, 15-20\% phosphor concentration samples showed the best balance between converted yellow light and non-converted blue light in both sphere $\mathrm{R}$ and sphere $\mathrm{T}$ : more concentrated samples avoid any blue light to be transmitted, while less concentrated samples $(<10 \%)$ yield less converted light.

\section{Conclusions}

We have presented the development of a robust instrument for the metrological evaluation of optical properties of solid samples or turbid media. Our choice for a double integrating sphere (DIS) system, instead of a single sphere system, was driven by the fact that with our set-up, absolute reflected and transmitted light from the sample can be simultaneously measured within the same experimental conditions and configuration. In this way, accurate measurements of absorption of blue incident light are available, together with the absolute fraction of reflected and transmitted light, providing valuable optical constants of the studied sample.

Our work has a special focus on the assessment of absolute photoluminescence quantum yield values for different phosphor families currently used in SSL. Our instrument is based on a custom-design double integrating sphere system where QY values are measured within 3\% uncertainty. Light losses within the optical system that generate important inaccuracies on the correct evaluation of photoluminescence quantum yields have been minimized. QY values obtained in our work are in fair agreement with values given by other authors; our values being slightly higher due to instrumental 
precision and/or different synthesis procedures carried out on the evaluated phosphors. The reliability and accuracy of our instrument was validated taking the YAG:Ce phosphor as reference, as its QY value was given by the supplier. Our biggest measurement imprecision comes from the spectrometer performance that can be affected by stray light or random variations of the dark current baseline (3\%) due to temperature changes within its environment. In addition, the advantages of using a laser source instead of a LED source to obtain accurate values of PL-QY with our DIS set-up have also been proven.

A Supercontinuum source will be soon implemented in our studies in order to better characterize the excitation spectra of these phosphors. Scattering properties of phosphors at non-absorbing wavelengths will also be available.

Competing interests

The authors declare that they have no competing interests.

\section{Authors' contributions}

PG implemented the double integrating sphere system for measuring the absolute photoluminescence quantum yield, made all the measurements presented in the publication and drafted the manuscript; MC and AG participated in the definition of the experimental set-up, made a critical analysis of the results and contributed to the finalization of the manuscript. All authors read and approved the final manuscript.

Received: 28 May 2014 Accepted: 23 December 2014

Published online: 05 February 2015

\section{References}

1. Seong TS, Han J, Amano H, Morkoç H. Packaging. Phosphors and White LED Packaging. In: Ascheron CE, Duhm AH, editors. III-Nitride Based Light Emitting Diodes and Applications. Heidelberg: Springer; 2013. p. 291-320

2. Liu Z, Liu S, Wang K, Luo X. Analysis of Factors Affecting Color Distribution of White LEDs. In: Proceedings of IEEE International Conference on Electronic Packaging Technology \& High Density Packaging (ICEPT-HDP 2008), Shanghai (China), 28-31 July 2008, E-ISBN: 978-1-4244-2740-6, doi:10.1109/ICEPT.2008.4607013.

3. Rohwer LS, Martin JE. Measuring quantum efficiencies of luminescent materials. J Lumin. 2005;115:77-90.

4. Murase N, Li C. Consistent determination of photoluminescence efficiency for phosphors in the form of solution, thin film and powder. J Lumin. 2008;128:1896-903.

5. Suzuki K, Kobayashi A, Kaneko S, Takehira K, Yoshihara T, Ishisda H, et al. Reevaluation of absolute luminescence quantum yields of standard solutions and a back-thinned CDD detector. Phys Chem Chem Phys. 2009;11:9850-60.

6. Würth C, Grabolle M, Pauli J, Spieles M, Resch-Genger U. Relative and absolute determination of fluorescence quantum yields of transparent samples. Nat Protoc. 2013:8:1535-50.

7. Smet PF, Parmentier AB, Poelman D. Selecting conversion phosphors for white light emitting diodes. J Electrochem Soc. 2011;158:R37-54.

8. Absolute PL quantum yield measurements system. http://www.hamamatsu.com/eu/en/product/category/5001/ 5009/5032/C9920-02/index.html

9. Liu Z, Liu S, Wang K, Luo X. Measurement and numerical studies of optical properties of YAG:Ce phosphor for white-emitting diode packaging. Appl Opt. 2010;49:247-57.

10. Zhu Y, Narendran N, Gu Y. Investigation of optical properties of YAG:Ce phosphor. Proc SPIE. 2006;6337:63370S.

11. Pickering JW, Prahl SA, Van Wieringen N, Beek JF, Sterenborg HJ, Gemert MJ. Double integrating sphere system for measuring the optical properties of tissue. Appl Opt. 1993;32:399-410.

12. Setlur A. Phosphors for LED-based solid state lighting. Electrochem Soc Interface. 2009;18:32-6.

13. Prahl S. Everything I think you should know about Inverse-Adding- Doubling. In: Manual on Inverse-AddingDoubling program. http://omlc.ogi.edu/software/iad/.

14. Suzuki K. Absolute photoluminescence quantum yield measurements by using an integrating sphere. New Orleans, LA, USA: Conference Phosphor Global Summit; 2013.

15. Xie RJ, Hintzen HT. Optical properties of oxy-nitride materials: a review. J Am Ceram Soc. 2013;96:665-87.

16. Jang MS, Kim WH, Kang YR, Song SB, Kim JP. Effect of particle size on the optical properties of yellow silicate phosphor in light emitting diodes. Int J Appl Ceram Technol. 2013;10:617-24.

17. Piquette AP, Hannah ME, Mishra KC. An investigation of self-absorption and corresponding spectral shift in phosphors. ECS Trans. 2012:41:1-9.

18. Keppens A, Zong Y, Podobedov VB, Nadal ME, Hanselaer P, Ohno Y. Fluorescence errors in integrating sphere measurements of remote phosphor type LED sources. In: Optical Measurement Systems for Industrial Inspection VII Book Series. Proc. SPIE 2011;8082, 808241.

19. Ahn TS, Al-Kaysi RO, Müller AM, Wentz KM, Bardeen CJ. Self-absorption correction for photoluminescence quantum yields from integrating sphere measurements. Rev Sci Instrum. 2007;78:086105. 
20. Bachmann V, Ronda C, Meijerink A. Temperature quenching of yellow $\mathrm{Ce}^{3+}$ luminescence on YAG:Ce phosphor. Chem Matter. 2009;21:2077.

21. Cho IH, Suh AD G, Lee JS, Yoo JS. On the stability and reliability of $\mathrm{Sr}_{1-x} \mathrm{Ba}_{x} \mathrm{Si}_{2} \mathrm{O}_{2} \mathrm{~N}_{2}: \mathrm{Eu}^{2+}$ phosphors for white LED applications. Opt Mater Express. 2012;2:1292-305.

22. Kim JS, Park YH, Choi JC, Park HL. Temperature-dependent emission spectrum of $\mathrm{Ba}_{3} \mathrm{MgSi}_{2} \mathrm{O}_{8}: \mathrm{Eu}^{2+}, \mathrm{Mn}^{2+}$ phosphor for white LED emitting diodes. Electrochem Solid-State Lett. 2005;8:H65-7.

Submit your manuscript to a SpringerOpen ${ }^{\circ}$ journal and benefit from:

- Convenient online submission

- Rigorous peer review

- Immediate publication on acceptance

- Open access: articles freely available online

- High visibility within the field

- Retaining the copyright to your article

Submit your next manuscript at $>$ springeropen.com 